\title{
HAUSDORFF DIMENSION AND BIACCESSIBILITY FOR POLYNOMIAL JULIA SETS
}

\author{
PHILIPP MEERKAMP AND DIERK SCHLEICHER
}

(Communicated by Bryna Kra)

\begin{abstract}
We investigate the set of biaccessible points for connected polynomial Julia sets of arbitrary degrees $d \geq 2$. We prove that the Hausdorff dimension of the set of external angles corresponding to biaccessible points is less than 1, unless the Julia set is an interval. This strengthens theorems of Stanislav Smirnov and Anna Zdunik: they proved that the same set of external angles has zero 1-dimensional measure.
\end{abstract}

\section{INTRODUCTION}

The filled-in Julia set $K$ of a polynomial $p: \mathbb{C} \rightarrow \mathbb{C}$, defined as the set of points in $\mathbb{C}$ with bounded orbits, is often an interesting set with rich topological, combinatorial or geometric properties. In many cases, such a set is a dendrite: a compact connected locally connected set that does not disconnect the plane. In some sense, such a set can often be viewed as an infinite tree. One way to ask our main question is, which proportion of this tree consists of "endpoints", and which proportion consists of "non-endpoints"? For finite (non-degenerate) trees, there are finitely many endpoints and a continuum of non-endpoints (on the arc); but what if the Julia set is a dendrite? What happens in more general cases of filled-in Julia sets? How are endpoints defined in general?

One way to make this definition precise is to say that a point $z$ in a tree is an endpoint unless there are points $x, y$ in the tree, different from $x$, such that $z$ is on an injective path connecting $x$ to $y$. This definition works best for path-connected Julia sets. The definition that we use is the following: a point $x \in K$ is an endpoint unless there are two curves in $\mathbb{C} \backslash K$, not homotopic to each other, that connect $\infty$ to $x$. Such points $x$ are also called biaccessible; an equivalent definition is that two different dynamic rays land at $x$ (see the next section). Our main result is that in almost all cases, most points in $K$ (in a very strong sense) are endpoints, unless $K$ is an interval (a straight line segment).

Theorem (Hausdorff dimension of biaccessible angles). Let $p$ be a polynomial of degree at least 2 with connected Julia set $J=\partial K$. Then the biaccessible points have external angles in a set of Hausdorff dimension less than 1, except when $J$ is an interval.

This result does not have any topological hypotheses on the Julia set other than that it be connected. It need not be locally connected or path connected or uniquely

Received by the editors April 14, 2011 and, in revised form, June 28, 2011.

2010 Mathematics Subject Classification. Primary 37F10, 37F20, 37F35.

Key words and phrases. Julia set, polynomial, biaccessible, Hausdorff dimension. 
path connected. One might thus wonder whether a similar statement might be true for planar dendrites (or even continua) with certain conformal self-similarity properties, whether arising in complex dynamics or elsewhere.

Our result extends known theorems by several people. The fact that biaccessible points have external angles in a set of 1-dimensional measure zero was shown by Smirnov 13 and Zdunik [17] (in other words, these points have zero harmonic measure), except if the Julia set is a straight line segment. Earlier, Zakeri [15] had shown this for quadratic polynomials with locally connected Julia sets.

The Hausdorff dimension of the set of external angles of biaccessible points has been investigated as well. Zakeri [16] estimated this dimension for certain real quadratic polynomials, and Bruin and Schleicher [4, Section 14], 5] gave estimates for all complex quadratic polynomials, as well as for certain subsets of the parameter space (the Mandelbrot set). Radu [11, in his bachelor's thesis, proved the same result as ours for the case of connected polynomial Julia sets satisfying certain additional hypotheses, including local connectivity of the Julia set and further assumptions on the critical values.

Remark 1.1. There are also some known lower bounds on the Hausdorff dimension of external angles of biaccessible points. The dimension is clearly zero for Julia sets with no or only countably many biaccessible points. Bruin and Schleicher [4, Section 14], [5] also showed that the dimension is zero for quadratic "Feigenbaum" polynomials: these are limits of Julia sets with only countably many biaccessible points. For all other quadratic polynomials with connected Julia sets, the dimension is strictly positive. It is natural to ask how these results extend to polynomials of higher degrees and whether the Hausdorff measure of the set of biaccessible angles in the "right" dimension is finite and positive (at least when the dimension is strictly positive).

Bruin and Schleicher [4, Section 14], 5] also showed that there can be no uniform upper bound on the Hausdorff dimension of biaccessible angles, even for fixed degrees: there are quadratic polynomials close to $z \mapsto z^{2}-2$, where the biaccessible points have Hausdorff dimension arbitrarily close to 1 .

Further properties of biaccessible points in polynomial Julia sets have been studied by Zakeri and Schleicher [12, 15].

Remark 1.2. The concept of biaccessibility is a topological one, defined in terms of homotopy classes of curves outside of the Julia set. However, all our arguments are combinatorial and would allow us to restate the result in a combinatorial way (that would actually be slightly stronger because not all dynamic rays must land). We will briefly discuss this in Section 6 .

\section{BACKGROUND}

Let $p: \mathbb{C} \rightarrow \mathbb{C}$ be a polynomial of degree $d \geq 2$, which we may as well assume to be monic. The filled Julia set of $p$ is the set of all points in $\mathbb{C}$ with bounded forward orbit under $p$, and the Julia set of $p$ is $J:=\partial K$. It satisfies $J=p(J)=p^{-1}(J)$, Lemma 4.3].

The sets $K$ and $J$ are non-empty compact subsets of $\mathbb{C}$. Moreover, $K$ is full, i.e., $\mathbb{C} \backslash K$ is connected. In this paper, we assume that $J$ (or equivalently $K$ ) is connected. In this case, there is a Riemann map

$$
\psi: \overline{\mathbb{C}} \backslash \overline{\mathbb{D}} \rightarrow \overline{\mathbb{C}} \backslash K \text {. }
$$


When $p$ is monic, then $\psi$ is unique by requiring $\psi(\infty)=\infty$ and $\lim _{z \rightarrow \infty} \psi(z) / z \rightarrow 1$. This Riemann map satisfies

$$
\psi\left(z^{d}\right)=p(\psi(z))
$$

i.e., it conjugates $p$ on its basin of $\infty$ to $z \mapsto z^{d}$.

For $t \in \mathbb{S}^{1}:=\mathbb{R} / \mathbb{Z}$, the image of the radial line $\left\{\psi\left(r e^{2 \pi i t}\right): r>1\right\}$ is called the dynamic ray at (external) angle $t$ and denoted $R_{t} \subset \overline{\mathbb{C}} \backslash K$; it satisfies $p\left(R_{t}\right)=R_{d t}$. Consider the radial limit

$$
\gamma(t)=\lim _{r \searrow 1} \psi\left(r e^{2 \pi i t}\right) .
$$

It need not exist for all $t \in \mathbb{S}^{1}$, but it is well known to exist for almost all $t$, 9 , Theorem 18.2]. If this limit exists, one says that the ray $R_{t}$ lands at the point $\gamma(t) \in J$.

For all angles $t \in \mathbb{S}^{1}$ for which $R_{t}$ lands, the ray $R_{d t}$ lands as well, and

$$
\gamma(d t)=p(\gamma(t)) .
$$

Thus $\gamma$ is a semiconjugation of multiplication by $d$ on $\mathbb{S}^{1}$ to the action of $p$ on the Julia set (restricted to those angles the rays of which land, and the corresponding landing points). In the particular case when the Julia set $J$ is locally connected, the map $\gamma: \mathbb{S}^{1} \rightarrow J$ is defined everywhere, and it is a continuous surjection $\gamma: \mathbb{S}^{1} \rightarrow J$ [9, Theorem 18.3].

We will denote distance on $\mathbb{S}^{1}$ by $\tau$ (normalized so as to inherit the metric locally from $\mathbb{R}$, and always measuring along the shorter of the two arcs connecting two points in $\left.\mathbb{S}^{1}\right)$. For an interval $I \subset \mathbb{S}^{1}$, let $\tau(I)$ denote its length.

A point $z \in J$ is called accessible if $z$ is the landing point of a dynamic ray (by Lindelöf's theorem, this is equivalent to the existence of a curve in $\mathbb{C} \backslash K$ converging to $z$ ). The point $z$ is called biaccessible if it is the landing point of at least two rays. If two dynamic rays $R_{t}$ and $R_{t^{\prime}}$ land together, we call the external angles $t$ and $t^{\prime}$ biaccessible angles, and we call $\left(t, t^{\prime}\right)$ a biaccessible angle pair. Let $\Lambda \subset \mathbb{S}^{1}$ be the set of all angles in biaccessible angle pairs. A ray pair is a set of two dynamic rays that land at a common point (so that their external angles form a biaccessible angle pair).

As usual, a critical point of $p$ is a point $z$ with $p^{\prime}(z)=0$, and $p(z)$ is the corresponding critical value.

Remark 2.1. Note that $z$ is biaccessible if and only if $p(z)$ is biaccessible, unless $z$ is a critical point for $p$. This also means that $t$ is a biaccessible angle if and only if so is $d t$, except when $R_{t}$ lands at a critical point.

Remark 2.2. It may also happen that three or more rays land at a single point. We call such a point "poly-accessible". It is well known and easy to see that such points always form a countable set [10, Proposition 2.18] (for every $\varepsilon>0$, there can only be finitely many landing points of three rays, the angles of which have mutual distance at least $\varepsilon$ ). If three or more rays land at the same point that is not eventually periodic and not eventually critical, then this landing point is called a "wandering triangle" (or, more generally, a "wandering polygon"), and the number of rays at such points, as well as the number of such orbits, satisfy certain bounds depending on the degree of $d$; in particular, these numbers are finite (compare [8, 2, 1]). If three or more rays land at a periodic point, then either the landing point is repelling or parabolic and the number of rays is finite or the landing point 
is a Cremer point (in which case it is not known whether any rays may land at all; compare [12). In total, there can thus be only countably many poly-accessible points, and except for Cremer points the total number of rays involved is countable.

\section{Endpoints of the Julia Set}

Definition 3.1 (J-Endpoint). A point $t \in \mathbb{S}^{1}$ will be called a J-endpoint of a Julia set if there exists a sequence $\left(t_{n}, t_{n}^{\prime}\right)$ of biaccessible angle pairs such that

$$
t_{n} \rightarrow t, \quad t_{n}^{\prime} \rightarrow t,
$$

and such that for all large $n$, the point $t$ lies in the shorter arc of $\mathbb{S}^{1}$ connecting $t_{n}$ and $t_{n}^{\prime}$.

Lemma 3.2 (Trichotomy). For every connected polynomial Julia set $J$, exactly one of the following three cases holds:

- $J$ has no biaccessible points and $\Lambda$ is empty;

- $J$ is an interval and $\Lambda$ is $\mathbb{S}^{1}$ minus two points;

- $J$ has at least three J-endpoints and $\Lambda$ is dense.

Proof. These three cases are clearly mutually exclusive. If $\Lambda$ is non-empty, then the preimage of any biaccessible point in $J$ is a biaccessible point, and it follows easily that $\Lambda$ is infinite and dense.

Now we show that if $\Lambda$ is non-empty, then $J$ has at least two J-endpoints. Consider a biaccessible angle pair $\left(t, t^{\prime}\right)$. The angles $t$ and $t^{\prime}$ separate the circle $\mathbb{S}^{1}$ into two open intervals, say $I$ and $I^{\prime}$. Since $\Lambda$ is dense, there is a biaccessible angle pair $\left(t_{1}, t_{1}^{\prime}\right)$ with $t_{1}, t_{1}^{\prime} \in I$. Let $I_{1} \subset I$ be the interval bounded by $t_{1}$ and $t_{1}^{\prime}$. It has strictly smaller length than $I$; in fact, choosing $t_{1}$ in the middle third of $I$, we can assure that the length of $I_{1}$ is at most $2 / 3$ of the length of $I$. Iterating this argument, we find a biaccessible angle pair in arbitrarily small intervals inside $I$ and hence at least one J-endpoint in $I$ (i.e., this J-endpoint is in the impression of a ray with angle in $I$ ). In a similar way we find at least one J-endpoint in $I^{\prime}$. Thus the Julia set $J$ has at least two J-endpoints.

If $J$ has exactly two J-endpoints, then $J$ is an interval by [17, Lemma 2] (and $p$ is a Chebyshev polynomial, up to sign); see also [18. (The idea is this: if a critical value is not a J-endpoint, then the corresponding critical point is a branch point of $J$ and creates extra J-endpoints; moreover, J-endpoints always map to J-endpoints. This implies that if $J$ has only two J-endpoints, then it is postcritically finite and has only two critical values. By conjugation, we may suppose that these two critical values are real. The polynomial $p$ has a Hubbard tree without branch points, and all critical values are endpoints of this tree. The Hubbard tree is thus backwards invariant, so it equals the Julia set.)

For us, the only interesting case is when the Julia set has at least three Jendpoints. We will assume this from now on.

\section{NARROW PREIMAGES OF RAY PAIRS}

Let $\left(t, t^{\prime}\right)$ be a biaccessible angle pair and $\left(t_{1}, t_{1}^{\prime}\right)$ one of its preimage biaccessible angle pairs, i.e., a biaccessible angle pair with $d t_{1}=t$ and $d t_{1}^{\prime}=t^{\prime}$. We call the preimage $\left(t_{1}, t_{1}^{\prime}\right)$ narrow if $\tau\left(t_{1}, t_{1}^{\prime}\right)=\tau\left(t, t^{\prime}\right) / d$. An (iterated) preimage biaccessible angle pair $\left(t_{n}, t_{n}^{\prime}\right)$ is called narrow of generation $n$ if $\left(t_{n}, t_{n}^{\prime}\right)$ is obtained from $\left(t, t^{\prime}\right)$ 
by taking $n$ generations of preimages, and all intermediate preimages are narrow (so that $\left.\tau\left(t_{n}, t_{n}^{\prime}\right)=\tau\left(t, t^{\prime}\right) / d^{n}\right)$.

Our assumption that the Julia set has at least three J-endpoints implies that there are three biaccessible angle pairs, say $\left(a, a^{\prime}\right),\left(b, b^{\prime}\right)$ and $\left(c, c^{\prime}\right)$, so that none of them separates the other two: we may assume that the cyclic order of these six angles is $a, a^{\prime}, b, b^{\prime}, c, c^{\prime}$. To simplify the reasoning, suppose that the longer of the two intervals of $\mathbb{S}^{1} \backslash\left\{a, a^{\prime}\right\}$ contains $\left\{b, b^{\prime}, c, c^{\prime}\right\}$, and similarly for $\mathbb{S}^{1} \backslash\left\{b, b^{\prime}\right\}$ and $\mathbb{S}^{1} \backslash\left\{c, c^{\prime}\right\}$. We will call biaccessible angle pairs with this property un-nested. Each Julia set with at least three J-endpoints clearly has three such angle pairs.

Lemma 4.1 (Number of narrow preimage biaccessible angle pairs). Consider a polynomial $p$ of degree $d \geq 2$ and suppose it has three un-nested biaccessible angle pairs. For generations $n \geq 0$, let $s_{n}$ be the combined number of narrow preimage biaccessible angle pairs of all three biaccessible angle pairs. Then $s_{n+1} \geq d s_{n}-$ $2(d-1)$.

Proof. Denote the three given biaccessible angle pairs by $\left(a, a^{\prime}\right),\left(b, b^{\prime}\right)$ and $\left(c, c^{\prime}\right)$. The set $\mathbb{C} \backslash \overline{R_{a} \cup R_{a^{\prime}}}$ consists of two components, say $B_{a}$ and $B_{a}^{\prime}$. The assumption that the three given ray pairs are non-nested means that one of these components, say $B_{a}^{\prime}$, contains the other two given ray pairs; define $B_{b}$ and $B_{c}$ analogously.

Suppose for simplicity that none of the ray pairs considered lands at a critical value. The immediate preimage of the biaccessible angle pair $\left(a, a^{\prime}\right)$ consists of $d$ disjoint biaccessible angle pairs that may or may not be nested. The corresponding ray pairs disconnect $\mathbb{C}$ into $d+1$ open complementary domains $U_{1}, \ldots, U_{d+1}$. Each of the $U_{j}$ maps under $p$ as a proper holomorphic map onto a component of $\mathbb{C} \backslash$ $\overline{R_{a} \cup R_{a^{\prime}}}$, so each $U_{j}$ has an associated mapping degree that equals the number of critical points on $U_{j}$ plus 1 (always counting multiplicities). A domain $U_{j}$ is narrow (i.e., $U_{j}$ is bounded by a single ray pair, and this ray pair is narrow) if and only if $U_{j}$ maps conformally onto $B_{a}$; this implies that $U_{j}$ does not contain a critical point.

The case with the maximal count of narrow components $U_{j}$ is when a single domain $U_{d+1}$ contains all $d-1$ critical points and maps onto $B_{a}^{\prime}$ with degree $d$, and all other domains $U_{1}, \ldots, U_{d}$ map conformally onto $B_{a}$ and are narrow.

If a domain $U_{j}$ contains a single critical point, then its mapping degree is 2 ; if $U_{j}$ maps to $B_{a}$, then the count of possible narrow domains $U_{j}$ decreases by 2 . Each further critical point in $U_{j}$ decreases this count by 1 . Therefore, the number of narrow components $U_{j}$ is at least $d$ minus twice the number of critical points the images of which are in $B_{a}$.

The same arguments apply to the angle pairs $\left(b, b^{\prime}\right)$ and $\left(c, c^{\prime}\right)$. Note that the domains $B_{a}, B_{b}$, and $B_{c}$ are disjoint, so each critical point that reduces the number of narrow components can count only for one of the three ray pairs. Therefore, the total number of narrow preimages of $\left(a, a^{\prime}\right),\left(b, b^{\prime}\right)$ and $\left(c, c^{\prime}\right)$ is $s_{1} \geq 3 d-2(d-1)=$ $d+2$. All $s_{1}$ ray pairs are narrow and non-nested. Note that initially we had $s_{0}=3$ angle pairs, and $s_{1} \geq d s_{0}-2(d-1)$, as claimed.

For the inductive step, the three initial angle pairs are replaced by $s_{n}$ non-nested ray pairs; for these, the argument can be repeated: each of these $s_{n}$ has at least $d s_{n}$ narrow preimages minus twice the number of critical points that map into the narrow complementary component of any ray pair, and again each critical point can reduce the count of narrow preimages for only one ray pair, and only by two. This yields the formula $s_{n+1} \geq d s_{n}-2(d-1)$, as claimed. 
Finally, if some ray pair lands at a critical value, then some of its preimage ray pairs merge, and the statement remains true for an appropriate choice of rays to form pairs.

Let $E$ be the set of external angles with the property that for each $t \in E$, there are infinitely many narrow biaccessible angle pairs $\left(t_{k}, t_{k}^{\prime}\right)$ on the backwards orbit of $\left(a, a^{\prime}\right),\left(b, b^{\prime}\right)$, or $\left(c, c^{\prime}\right)$ so that the interval $\left(t_{k}, t_{k}^{\prime}\right) \subset \mathbb{S}^{1}$ contains $t$, and so that the lengths of these intervals tend to 0. ( $E$ stands for "endpoints" of the Julia set.)

Lemma 4.2. Angles in $E$ are not part of any biaccessible angle pair.

Proof. Suppose that $\left(t, t^{\prime}\right)$ is a biaccessible angle pair, but $t \in E$. Then there is a narrow biaccessible angle pair $\left(t_{k}, t_{k}^{\prime}\right)$ with $\tau\left(t_{k}, t_{k}^{\prime}\right)<\tau\left(t, t^{\prime}\right)$ and so that the interval $\left(t_{k}, t_{k}^{\prime}\right)$ contains $t$; this interval thus cannot contain $t^{\prime}$. This is a contradiction unless all the rays at angles $t_{k}, t_{k}^{\prime}, t, t^{\prime}$ land at the same point. This would imply that for all biaccessible angle pairs $\left(t_{m}, t_{m}^{\prime}\right)$ of higher generation than $\left(t_{k}, t_{k}^{\prime}\right)$ such that $t$ is contained in the interval $\left(t_{m}, t_{m}^{\prime}\right)$, the rays $R_{t_{m}}$ and $R_{t_{m}^{\prime}}$ must also land at the same point, so infinitely many rays would land at this point. We will now show that this is impossible.

Indeed, the set $\mathbb{C} \backslash \overline{R_{t_{k}} \cup R_{t_{k}^{\prime}}}$ consists of two components; let $U_{k}$ be the component containing $R_{t}$, and define $U_{m}$ similarly. We may assume that both angle pairs are on the backwards orbit of the same angle pair $\left(a, a^{\prime}\right),\left(b, b^{\prime}\right)$, or $\left(c, c^{\prime}\right)$, and indeed that $\left(t_{m}, t_{m}^{\prime}\right)$ is on the backwards orbit of $\left(t_{k}, t_{k}^{\prime}\right)$, say after $s>0$ iterations. Since all biaccessible angle pairs are narrow, this implies that $p^{o s}: U_{m} \rightarrow U_{k} \supset U_{m}$ is a conformal isomorphism with conformal inverse $q: U_{k} \rightarrow U_{m}$. Iterating $q$, it follows that $t$ is a periodic angle, so the landing point is repelling or parabolic, and only finitely many rays land there (compare Remark 2.2).

So all we need to do is prove that $\mathbb{S}^{1} \backslash E$ has Hausdorff dimension less than 1 (most angles correspond to J-endpoints). We will prove the following result.

Lemma 4.3. The Hausdorff dimension of $\mathbb{S}^{1} \backslash E$ satisfies $\operatorname{dim}_{H}\left(\mathbb{S}^{1} \backslash E\right)<\eta<1$, where $\eta$ depends only on $d$ and the lengths of $\left(a, a^{\prime}\right),\left(b, b^{\prime}\right)$, and $\left(c, c^{\prime}\right)$.

Remark 4.4. Let $\alpha$ be the minimum of the three lengths of $\left(a, a^{\prime}\right),\left(b, b^{\prime}\right),\left(c, c^{\prime}\right)$. The quantity $\alpha$ is, in some sense, a measure of the "size" of branch points of the Julia set: in a locally connected Julia set, a branch point $q$ is the landing point of at least three dynamic rays, and its size can be defined as

$$
\begin{gathered}
s(q)=\sup \left\{\delta>0 \text { : three rays at angles } t_{1}, t_{2}, t_{3} \text { land at } q\right. \\
\text { with } \left.\tau\left(t_{i}, t_{j}\right)>\delta \text { for } i \neq j\right\} .
\end{gathered}
$$

This definition coincides with the maximal possible value of $\alpha$ in the locally connected case, so $\alpha$ can indeed be seen as a measure of size of a branch point (a branch point is "small" if all the rays landing at it can be grouped into two sets so that the angles are contained in short intervals).

The proof of Lemma 4.3 will be given, in somewhat abstract form, in the next section. 


\section{HAUSDORFF DIMENSION}

Let $g: \mathbb{S}^{1} \rightarrow \mathbb{S}^{1}$ be multiplication of angles by $d \geq 2$.

Lemma 5.1. Let $I_{1}, I_{2}, I_{3} \subset \mathbb{S}^{1}$ be three disjoint open intervals, each of length $d^{-N}$ for some $N \in \mathbb{N}$, and each bounded by endpoints in $\mathbb{Z} / d^{N}$ (projected into $\mathbb{R} / Z$ ). Let $\mathcal{I}_{0}:=\left\{I_{1}, I_{2}, I_{3}\right\}$. For $n \geq 1$, let $\mathcal{I}_{n}$ be the set of all g-preimages of all intervals in $\mathcal{I}_{n-1}$, except that $2(d-1)$ intervals are missing in $\mathcal{I}_{n}$. Then $\left|\mathcal{I}_{n}\right|=d^{n}+2$, and for every $m \in \mathbb{N}$, the set

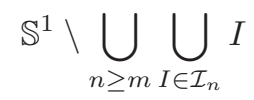

has Minkowski dimension at most $1-3 / d^{N} N \log d<1$.

Proof. We have $\left|\mathcal{I}_{0}\right|=3=d^{0}+2$ and $\left|\mathcal{I}_{n+1}\right|=d\left|\mathcal{I}_{n}\right|-2(d-1)=d^{n+1}+2$ by induction.

The condition on the endpoints assures that whenever $n^{\prime}>n$, each interval in $\mathcal{I}_{n^{\prime}}$ is either disjoint from or contained in any interval in $\mathcal{I}_{n}$.

For $n \in \mathbb{N}$, set $A_{n}:=\bigcup_{i \in \mathcal{I}_{n}} I$, and let $c_{n}$ be the number of intervals of length $d^{-n}$ required to cover $\mathbb{S}^{1} \backslash A_{n}$.

The set $\mathbb{S}^{1} \backslash A_{0}$ consists of 3 intervals of length less than 1 . The set $\mathbb{S}^{1} \backslash g^{-n}\left(A_{0}\right)$ thus consists of $3 d^{n}$ intervals of length less than $d^{-n}$, for each $n$ (in this argument, we do not delete the $2(d-1)$ intervals in each generation).

In general, we have $c_{n+1} \leq d c_{n}+2(d-2)$ : taking preimages under $g$ increases the required number of intervals by a factor of $d$ (the length of the intervals decreases by a factor $d$ ), and each removed interval in $\mathcal{I}_{n+1}$ might require an extra covering interval.

Choose $\beta>0$ so that $\gamma:=(1+\beta)^{N}\left(1-3 d^{-N}\right)<1$. There is an $M \in \mathbb{N}$ so that $c_{n+1} \leq(1+\beta) d c_{n}$ for all $n \geq M$; possibly by enlarging $M$, suppose that $M$ is divisible by $N$.

The set $\mathbb{S}^{1} \backslash A_{M}$ can be covered by some number $C:=c_{M}$ of intervals of length $d^{-M}$. Then $\mathbb{S}^{1} \backslash A_{M+N}$ can be covered by $c_{M+N} \leq d^{N}(1+\beta)^{N} C$ intervals of length $d^{-(M+N)}$, and $\mathbb{S}^{1} \backslash\left(A_{M+N} \cup A_{0}\right)$ can be covered by $\left(d^{N}-3\right)(1+\beta)^{N} C=\gamma d^{N} C$ intervals of the same length, because one in $d^{N}$ intervals is contained in each of the intervals in $A_{0}$.

This argument can be repeated: $\mathbb{S}^{1} \backslash\left(A_{M+2 N} \cup A_{N}\right)$ can be covered by $(1+$ $\beta)^{N} \gamma d^{2 N} C$ intervals of length $d^{-(M+2 N)}$, and for $\mathbb{S}^{1} \backslash\left(A_{M+2 N} \cup A_{N} \cup A_{0}\right)$, at most $\left(1-3 d^{-N}\right)(1+\beta)^{N} \gamma d^{2 N} C=\gamma^{2} d^{2 N} C$ intervals are required.

Let us focus on the case $m=0$ in the claim: we are interested in the set $\mathbb{S}^{1} \backslash \bigcup_{n \geq 0} A_{n}$, and this is contained in

$$
\mathbb{S}^{1} \backslash \bigcup_{k \geq 0} A_{k N} \subset \mathbb{S}^{1} \backslash\left(A_{M+K N} \cup A_{(K-1) N} \cup A_{(K-2) N} \cup \cdots \cup A_{N} \cup A_{0}\right),
$$

and this latter set can be covered by $\gamma^{K} d^{K N} C$ intervals of length $d^{-(M+K N)}$.

For the Minkowski dimension, we get the upper bound

$$
\lim _{K \rightarrow \infty} \frac{\log \left(\gamma^{K} d^{K N} C\right)}{\log d^{M+K N}}=\frac{\log \left(\gamma d^{N}\right)}{\log d^{N}}=1+\frac{\log \left(1-3 d^{-N}\right)+N \log (1+\beta)}{\log d^{N}} .
$$

Now $\beta$ can be chosen arbitrarily close to 0 . Since $\log \left(1-3 d^{-N}\right)<-3 d^{-N}$, the dimension is bounded above by $1-3 d^{-N} / N \log d$. 
Now we treat the case $m>0$. The set $\mathbb{S}^{1} \backslash \bigcup_{n \geq 0} A_{n}$ can be covered by $\gamma^{K} d^{K N} C$ intervals of length $d^{-M+K N}$, for every $K \geq 0$, so $\mathbb{S}^{1} \backslash \bigcup_{n \geq m} A_{n}$ can be covered by $(1+\beta)^{m} \gamma^{K} d^{K N+m} C$ intervals of length $d^{-(M+K N+m)}$, and this leads to the same dimension.

Corollary 5.2. Using the notation of the previous lemma, the set

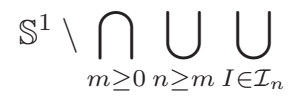

has Hausdorff dimension at most $1-3 / d^{N} N \log d$.

Proof. By the lemma, each set $B_{m}:=\mathbb{S}^{1} \backslash \bigcup_{n \geq m} A_{n}=\mathbb{S}^{1} \backslash \bigcup_{n \geq m} \bigcup_{I \in \mathcal{I}_{n}} I$ satisfies the same upper bound for Minkowski dimension, hence Hausdorff dimension. The set we are interested in is $\mathbb{S}^{1} \backslash \bigcap_{m} \bigcup_{n \geq m} A_{m}=\bigcup_{m}\left(\mathbb{S}^{1} \backslash \bigcup_{n \geq m} A_{n}\right)=\bigcup_{m} B_{m}$, and Hausdorff dimension is stable under countable unions.

Proof of Lemma 4.3. If the Julia set has three J-endpoints, then there are three biaccessible angle pairs $\left(a, a^{\prime}\right),\left(b, b^{\prime}\right),\left(c, c^{\prime}\right)$ so that the three intervals $\left(a, a^{\prime}\right)$, $\left(b, b^{\prime}\right),\left(c, c^{\prime}\right)$ (as subsets of $\mathbb{S}^{1}$ ) are disjoint. Let $\alpha>0$ be the minimum of their lengths. Their combined number of narrow preimages of generation 1 is at least $3 d-2(d-2)=d+2$ by Lemma 4.1. If $d \geq 5$, then at least one of these three intervals, say $\left(a, a^{\prime}\right)$, has three narrow preimages of length $\alpha / d$.

Call these intervals $I_{1}, I_{2}, I_{3}$. The number of narrow preimages of further generations grows as in Lemma 4.1. If we construct sets of intervals $\mathcal{I}_{n}$ as above and set $A_{n}:=\bigcup_{I \in \mathcal{I}_{n}} I$, then we have $E=\bigcap_{m \geq 0} \bigcup_{n \geq m} A_{n}$.

Our intervals do not yet satisfy the condition on the form of the endpoints, so we cannot directly apply the corollary. Restricting $\left(a, a^{\prime}\right)$ to a subinterval of length at least $1 /(2 d)$ times the original length, we obtain an interval $I_{0} \subset\left(a, a^{\prime}\right)$ that is bounded by two numbers $k / d^{N}$ and $(k+1) / d^{N}$ for $k, N \in \mathbb{N}$. This yields smaller sets $A_{n}$ and a smaller set $E$, hence a larger set $\mathbb{S}^{1} \backslash E$. The corollary applied to this larger set shows that $\mathbb{S}^{1} \backslash E$ has Hausdorff dimension less than 1.

If $d \in\{3,4\}$, then we might have to resort to narrow intervals of generation two, of which there are at least $d^{2}+2 \geq 11$ for all three intervals combined, and the argument proceeds as above (except that the dimension formula uses intervals of length $\alpha / d^{2}$, rather than $\left.\alpha / d\right)$.

Finally, if $d=2$, then we have to go one generation further.

This also proves the theorem.

\section{A REMARK ON LAMinATIONS AND ENTROPY}

As mentioned earlier, our results can also be stated in terms of laminations of the Julia set, as developed by Thurston [14, Chapter II]. In the complex unit disk $\mathbb{D}$, we identify the boundary $\partial \mathbb{D}=\mathbb{S}^{1} / \mathbb{Z}$ with the set of external angles. For a given connected polynomial Julia set $J$, we connect two angles $\alpha, \beta \in \partial \mathbb{D}$ by a geodesic of $\mathbb{D}$ whenever the two rays at angles $\alpha$ and $\beta$ land at a common (biaccessible) point. One usually uses hyperbolic geodesics for this purpose because this yields clearer pictures, even though the results are equivalent. Every biaccessible point is thus represented by an arc in $\mathbb{D}$; poly-accessible points are represented by polygons. The lamination $J$ is the closure of all these arcs (if the Julia set is locally connected, 

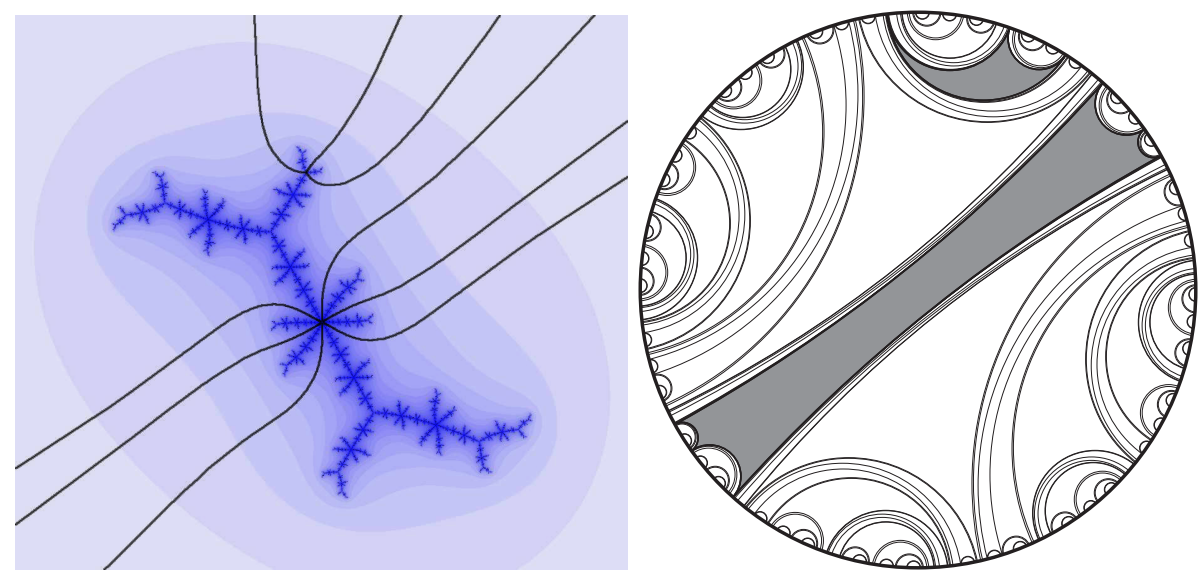

FiguRE 1. Left: a quadratic polynomial with three rays landing at the critical point, and thus six rays landing at the critical value. Right: a lamination modeling this Julia set. The critical point corresponds to a hexagon, the critical value to a triangle (both shaded).

then the set of arcs is closed to begin with), and each arc is called a leaf in this lamination. A degenerate leaf is one that connects an angle to itself (it is a point).

All our arguments, especially those in the key Section 4 are combinatorial in nature and can thus be stated entirely in terms of Julia set laminations or, more combinatorially, in terms of abstract laminations that satisfy certain natural invariance properties as discussed in [14, Chapter II] (for the relation of laminations to Julia sets, see the appendix in [14]). We thus obtain an estimate about the Hausdorff dimension of the set of endpoints of non-degenerate leaves in an invariant lamination. This setting relates to recent work of Thurston (as communicated in various talks and personal communications) as follows: if this dimension of endpoints is $\eta$, then the union of the leaves has dimension $1+\eta$. This dimension is related to the core entropy of a postcritically finite Julia set: such a Julia set has a Hubbard tree (defined as a minimal invariant tree connecting all postcritical points), and the core entropy is the entropy of the polynomial restricted to the Hubbard tree. If $H$ denotes the core entropy, then $\eta=H / \log d$. Thurston's formula thus relates the topological concept of biaccessible points, and more precisely the geometric concept of the Hausdorff dimension of their angles, to the dynamic concept of entropy on the Hubbard tree.

\section{ACKNOWLEDGEMENTS}

The authors would like to thank Remus Radu, Saeed Zakeri and especially Xavier Buff for useful discussions on this topic and on an earlier draft of this paper.

\section{REFERENCES}

1. A. Blokh, C. Curry, G. Levin, L. Oversteegen, D. Schleicher, An extended Fatou-Shishikura inequality and wandering branch continua for polynomials. Manuscript, submitted.

2. A. Blokh, G. Levin, An inequality for laminations, Julia sets and "growing trees". Ergodic Theory Dynam. Systems 221 (2002), 63-97. MR1889565(2003i:37045) 
3. H. Bruin, A. Kaff, D. Schleicher, Symbolic dynamics of quadratic polynomials. Monograph, in preparation.

4. H. Bruin, D. Schleicher, Symbolic dynamics of quadratic polynomials, Mittag Leffler Preprint 7 (2001/02). To appear as [3].

5. H. Bruin, D. Schleicher, Hausdorff dimension of biaccessible angles for quadratic polynomials. Manuscript, in preparation.

6. A. Douady, J. H. Hubbard, Étude dynamique des polynômes complexes, I, Publ. Math. Orsay, 1984. MR0762431 (87f:58072a)

7. K. Falconer, Fractal geometry. Mathematical foundations and applications, 2nd edn., Wiley, 2003. MR2118797 (2006b:28001)

8. J. Kiwi, Wandering orbit portraits. Trans. Amer. Math. Soc. 3544 (2002), 1473-1485. MR:1873015 (2002h:37070)

9. J. Milnor, Dynamics in one complex variable, 3rd edn., Princeton University Press, 2006. MR2193309 (2006g:37070)

10. C. Pommerenke, Boundary behaviour of conformal maps, Springer, 1992. MR.1217706 (95b:30008)

11. R. Radu, Hausdorff dimension and biaccessibility for polynomial Julia sets. Bachelor's thesis, Jacobs University, 2007.

12. D. Schleicher, S. Zakeri, On biaccessible points in the Julia set of a Cremer quadratic polynomial. Proc. AMS 1283 (2000), 933-937. MR1637424(2000e:37057)

13. S. Smirnov, On supports of dynamical laminations and biaccessible points in polynomial Julia sets, Colloq. Math. 87 (2001) 2, 287-295. MR.1814670(2001m:37092)

14. W. Thurston, On the geometry and dynamics of iterated rational maps. In: D. Schleicher (ed.), Complex dynamics: families and friends, A K Peters, Wellesley, MA, 2009, 1-137. MR2508255 (2010m:37076)

15. S. Zakeri, Biaccessibility in quadratic Julia sets, Ergod. Th. \& Dynam. Sys. 20 (2000), 18591883. MR1804961 (2001k:37068)

16. S. Zakeri, External rays and the real slice of the Mandelbrot set, Ergod. Th. \& Dynam. Sys. 23 (2003), 637-660. MR 1972243 (2004a:37057)

17. A. Zdunik, On biaccessible points in Julia sets of polynomials, Fund. Math. 163 (2000), 277-286. MR1758329(2001f:37058)

18. A. Zdunik, Parabolic orbifolds and the dimension of the maximal measure for rational maps, Invent. Math. 99 (1990), 627-649. MR.1032883(90m:58120)

Department of Mathematics, Malott Hall, Cornell University, Ithaca, New York 14853-4201

E-mail address: pmeerkamp@math.cornell.edu

School of Engineering and Science, Jacobs University, Postfach 750 561, D-28725

Bremen, Germany

E-mail address: dierk@jacobs-university.de 